\title{
A THERAPEUTIC POTENTIAL OF NICOTINE: REASSESSING THE CURRENT PARADIGM OF NICOTINE PHARMACOTHERAPY, LITERATURE REVIEW
}

\author{
MARCIN DELIJEWSKI ${ }^{1}$, LEON KOŚMIDER ${ }^{2}$, RADOSŁAW BALWIERZ ${ }^{3 *}$, MEGAN TOMAS $^{4}$, \\ DANIEL YI ${ }^{5}$, BARTOSZ KOSZOWSKI ${ }^{6}$, DOMINIK MARCINIAK ${ }^{7}$ and BOŻENA KAROLEWICZ
}

'Department of Pharmacology, School of Medicine with the Division of Dentistry in Zabrze, Medical University of Silesia, Poland

${ }^{2}$ Department of General and Analytical Chemistry, School of Pharmacy with the Division of Laboratory

Medicine in Sosnowiec, Medical University of Silesia, Poland

${ }^{3}$ Silesian Medical College in Katowice, Mickiewicza 29, Katowice 40-085, Poland

${ }^{4}$ Notre Dame of Maryland University, School of Pharmacy, 4701 North Charles St, Baltimore, MD 21210, USA

${ }^{5}$ University of Maryland School of Pharmacy, Baltimore, 20 North Pine Street, Baltimore, MD 21201, USA

${ }^{6}$ Battelle Public Health Center for Tobacco Research, Baltimore Health and Analytics, 6115 Falls Road, Suite 200, Baltimore, MD 21209, USA

${ }^{7}$ Wroclaw Medical University, Department and Institute of Pharmaceutical Technology, Wroclaw, Borowska 211, Poland

\begin{abstract}
Nicotine is a widely known alkaloid synthesized from tobacco plants, being the main constituent of tobacco smoke and cigarettes. Nicotine has also gained eminence as the therapeutic option in managing smoking cessation and even other health conditions. However, the therapeutic potential of nicotine in other diseases has yet to be completely assessed. This information void stems from an inherent aversion from researchers in assessing nicotine's risk-benefit, due to its toxicities. We present information on the current body of evidence relating to non-traditional therapeutic applications of nicotine to fill this literature void. The purpose of this work is to present the current literature on the therapeutic uses of nicotine in treating various diseases. Electronic search in PubMed for relevant research relating to the therapeutic potential of nicotine in various diseases. The therapeutic significance of nicotine is used in the nicotine replacement therapy. Research shows that nicotine may have other therapeutic applications in some diseases. We discussed an application of nicotine in the diminished prevalence of Parkinson's disease, decreasing symptoms of Tourette's syndrome, psychiatric diseases like schizophrenia, depression, and management of pain. We also reviewed nicotine dosing, type of formulations, and compliance which are crucial factors in the therapeutic applications of nicotine.
\end{abstract}

Keywords: nicotine, therapeutic potential, Parkinson's disease, Alzheimer's disease, schizophrenia, Tourette's syndrome, pain management, ulcerative colitis

Nicotine is an alkaloid synthesized by tobacco plants. Use of nicotine is highly prevalent due to its high addictiveness. The most popular way of nicotine use is cigarette smoking (1). Nicotine has also gained therapeutic significance, for example as an active compound in nicotine replacement therapy (NRT). Research shows nicotine may have other therapeutic applications in some diseases. However, due to the stigma associated with the negative consequences of smoking, not much attention has been paid by the scientific community to investigate this therapeutic role of nicotine.
Nicotine is absorbed from cigarette smoke, electronic cigarettes, nicotine gums, inhalers, nasal sprays, sublingual tablets and transdermal patches (1). The absorption can be through lungs, oral mucosa, and skin. The most effective way of nicotine delivery is tobacco smoke, which delivers nicotine directly to the lungs. Absorption of nicotine in the lungs provides the most rapid delivery to the brain (2). When smoked, this process begins with the vaporization of nicotine after inhalation of tobacco smoke (conventional cigarettes) or nicotine aerosol (electronic cigarette). Subsequently, the

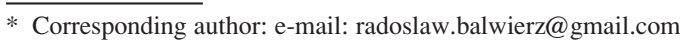


vaporized nicotine is rapidly absorbed in the lungs, passing through the circulatory system and eventually reaches the brain.

Nicotinic receptors are ligand-gated ion channels which have a pentameric structure, formed by eleven subunits (eight $\alpha$ and three $\beta$ units) identified in mammalians (3). Each subunit has a-transmembrane spanning region (3). The receptor protein contains binding sites for nicotine and acetylcholine on the side exposed to the synapse. The receptor also contains an ion channel for $\mathrm{Na}^{+}$and $\mathrm{K}^{+}$in its membrane (4). The acetylcholine receptors are involved in many brain functions that can be affected by major diseases in humans. The high diversity of the nicotinic receptors is caused by various combinations of 17 genes encoding subunits of these receptors (5). Because nicotine acts through nicotine acetylcholinergic receptors that have a complex structure, nicotine has a wide spectrum of biological effects.

Nicotine mediates the release of multiple neurotransmitters - the most important being dopamine (DA). In the brain, nicotine reveals the reinforcement effect, related to dopamine release from DA neurons (1).

Dopamine is associated with a variety of functions, one of which plays a major role in rewardrelated behavior. Due to these dopaminergic properties, a pleasurable euphoric state is experienced, which is one of the many reasons nicotine becomes addictive and, thus, recognized as an addictive drug $(2,6)$.
Nicotine acts as an agonist at most nicotinic acetylcholine receptors (nAChRs), except two nicotinic receptor subunits (nAChR $\alpha 9$ and $\mathrm{nAChR} \alpha 10$ ) where it acts as a receptor antagonist (7). The halflife of nicotine in the human body is about $2 \mathrm{~h}(8)$. Nicotine is metabolized mainly in the liver by cytochrome P450 2A6 (CYP2A6) (9) and about 70$80 \%$ of the absorbed nicotine is metabolized to cotinine. The major metabolites of cotinine are cotinine glucuronide, trans-3'-hydroxycotinine, 5'-hydroxycotinine, nicotine $\mathrm{N}^{\prime}$-oxide and nornicotine (7). Other CYP 450 isoforms are involved in the metabolism of nicotine. These are CYP2B6, CYP2D6, and CYP2E1 (10), however, CYP2A6 is mainly responsible for the oxidation of nicotine to cotinine. Among the various known side effects of nicotine; addiction, dependence, tolerance, and withdrawal have been the most prevalent issues. Tolerance develops due to an increase in nAChRs in the brain causing a decrease in the effect of nicotine as the user becomes less naive. Once a user becomes tolerant, dependence and addiction may develop, leading to potential nicotine abuse (2). Prior to and after the landmark publication about nicotine by the U.S. Surgeon General in 1964 , there have been numerous studies indicating the deleterious effects of nicotine (11).

Many compounds in combustible and noncombustible tobacco products have significant interactions with various medications and some are highly toxic or carcinogenic. As a result, nicotine has not been broadly investigated as a potential therapeutic agent. However, several recently published studies

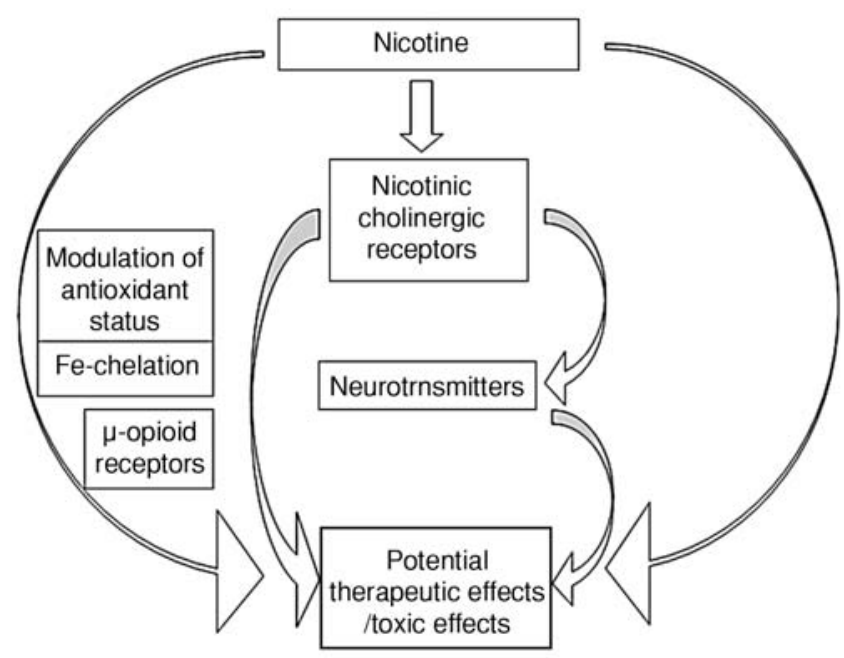

Figure 1. Complex effect of nicotine on the human body 


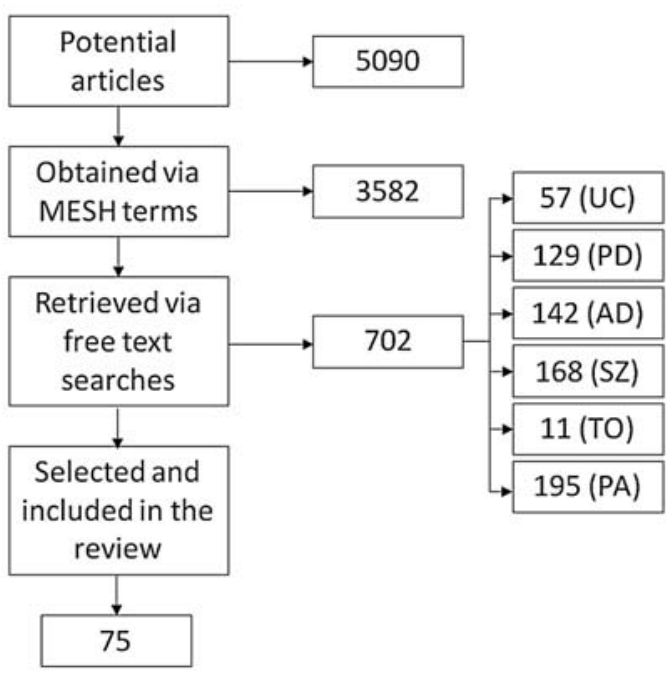

Figure 2. Consort diagram

have shown nicotine to be a safe and effective drug when controlled and dosed appropriately (12-15). This has prompted a renewed interest in nicotine as a potential treatment option for diseases other than nicotine addiction-ulcerative colitis, Parkinson's disease, Alzheimer's disease, schizophrenia, Tourette's syndrome, and pain management. Nicotine delivery systems that have reached the market in recent years as over the counter products - electronic cigarettes, gums, lozenges, sublingual tablets, and transdermal patches - may potentially serve in a therapeutic role (16).

In elucidating the mechanism underlying the therapeutic effects of nicotine, pharmacological studies involving nicotine and nicotinomimetics have been used to study nAChRs in the brain, their roles in various disease states, as well as other potential mechanisms of action of nicotine (Fig. 1).

In this paper, we reviewed and assessed the therapeutic validity of nicotine based on the evidence available in the current scientific literature.

\section{METHODS}

We searched the PubMed database for relevant trials using a combination of MeSH terms ("Nicotine/administration and dosage"[Mesh] OR "Nicotine/adverse effects"[Mesh] OR "Nicotine/agonists"[Mesh] OR "Nicotine/analogs and derivatives"[Mesh] OR "Nicotine/analysis"[Mesh] OR "Nicotine/pharmacology"[Mesh] OR "Nicotine/therapeutic use"[Mesh] OR "Nicotine/therapy”[Mesh]) and free text ('nicotine ulcerative colitis', 'nicotine Alzheimer's disease', 'nicotine Parkinson's disease', 'nicotine schizophrenia', 'nicotine Tourette's', and 'nicotine pain'. We searched for articles mainly from 2005-2015 that were in English and involved humans.

\section{RESULTS}

The literature search produced a total of 5090 potential articles. Of these articles, 3582 were obtained via MeSH terms while 702 articles were retrieved via free text searches [57 (Ulcerative colitis, UC), 129 (Parkinson's disease, PD), 142 (Alzheimer's disease, AD), 168 (Schizophrenia, SZ), 11 (Tourette's, TO), 195 (Pain, PA)]. After scanning the abstract and titles for relevance, 75 of the initial 5090 articles were selected and included in the review. The final review includes 48 articles from 2005-2015, 1 article from 1981, 1 article from 1985, 1 article from 1992, 1 article from 1994, 2 articles from 1995, 1 article from 1996, 1 article from 1997, 2 articles from 1998, 5 articles from 1999, 4 articles from 2000, 2 articles from 2001, 2 articles from 2002, 2 articles from 2003 and 2 articles from 2004. Additional information is included on the Consort diagram (Fig. 2).

\section{Use of nicotine in treatment of tobacco addiction}

In the Anatomical Therapeutic Chemical (ATC) classification nicotine is categorized as a smoking cessation drug used in the treatment of addictions (N $07 \mathrm{BA}$; nicotine replacement therapy). NRT includes nicotine gums, inhalers, nasal sprays, sublingual tablets, lozenges, and transdermal patches. The concept of using nicotine for smoking cessation derived from the observations that in addicted people the lack of nicotine causes withdrawal symptoms which force them to seek another dose of nicotine (e.g. smoking another cigarette). Delivery of pure nicotine to the organism relieves the withdrawal symptoms. Pharmaceutical formulations of nicotine with high bioavailability, reaching up to 75$100 \%$ for transdermal patch (7), $60-80 \%$ for nasal spray (7), 55-78\% for gum (7) and $65 \%$ for sublingual tablets (7), are good sources of pure nicotine which mimics the effect of tobacco smoke. Compared to conventional cigarettes, NRTs are better controlled, in the sense that each dose of nicotine is precisely delivered at a constant rate, and they do not deliver toxins associated with combustion process (16). However, NTR effectiveness is limited by slow absorption rate and low systemic delivery of nicotine $(16,17)$. Findings for the effective- 
ness of NRT versus placebo are mixed, while some studies report no difference (18) and one study reports higher rates of abstinence for NRT users (the rate of quitting increases from 50 to $70 \%$ ) (19). The comparison of the effectiveness of varenicline, a drug used for smoking cessation, to NRT, reported higher quitting rates in the varenicline group (20). Therefore, NRT may not be an effective treatment for all patients.

\section{Role in neurodegenerative diseases Parkinson's disease}

Parkinson's disease is a neurodegenerative disorder characterized by dopaminergic neuronal cell loss in the substantia nigra pars compacta; the continuous loss of dopaminergic neurons has been attributed to their vulnerability to oxidative stress $(21,22)$. In addition, it has been suggested that the metabolism of dopamine also contributes to the oxidative stress resulting in changes to mitochondrial cell function (23). Clinically, the most common manifestations of PD are resting tremor, rigidity, bradykinesia, and postural instability $(24,25)$.

The predicted therapeutic potential of nicotine in PD stems from epidemiological studies which showed smokers to have lower rates of neurodegenerative disorders than non-smokers (26). The prevalence of PD has been observed to be lower in smokers than in non-smokers and a dose-response relationship exists (27-32).

An analysis of longitudinal data from over 300,000 participants of the NIH-AARP (formerly known as American Association of Retired Persons) Diet and Health (DH) Study showed that higher smoking duration and fewer years since smoking cessation was associated with decreased odds of developing PD (27).

Although the detailed mechanism of neuroprotection is unclear, several theories suggest that the activation of nAChRs by nicotine might underlie this effect (26) so nicotine has been suggested to have pro-cognitive and neuroprotective effects for PD (33).

Nicotine is understood to have a receptor mediated and antioxidative mechanism of action in PD (26). As a nAChR agonist, nicotine increases levels of dopamine within the central nervous system, an effect that mimics the action of current treatments for PD such as L-DOPA which alleviates the symptoms of neurodegeneration (34). Nicotine, as an agonist of the nAChR's, can theoretically lead to upregulation of $\mathrm{Bcl}-2$ and $\mathrm{Bcl}-\mathrm{x}$, which are the antiapoptotic proteins, which may result in prevention of neuronal death induced by neurotoxins, such as rotenone and glutamate (35). The molecular mecha- nism of nicotine action involving the role of nicotinic receptors may be more significant as the nicotinic receptor containing beta 2 -subunit serves as a critical link between the release of acetylcholine and the dopaminergic reward (5).

Another mechanism that may be responsible for the therapeutic role of nicotine in PD is its ability to bind with free iron. Free iron is known to possess a degenerative role in neurodegenerative diseases through the Fenton reaction, nicotine seems to inhibit this process, most likely, by binding iron. Furthermore, nicotine metabolites may have more enhanced chelating abilities than nicotine itself (34, 36).

Research published by Quik et al. (37) indicate that pre-treatment with nicotine protects the mesencephalic neurons in cells culture against dopaminergic nigral neural degeneration by dopaminergic neurotoxins. Similar results from Takeuchi et al. (38) indicate that nicotinic neuroprotection is via nAChRs and that both $\alpha 7$ and $\alpha 4 \beta 2$ receptors may have relationships with neuroprotection. Additionally, glial cells may be partly responsible for nicotinic neuroprotection. Stimulation of microglial cells, which contain $\alpha 7 \mathrm{nAChRs}$, reduces the release of cytotoxic cytokines such as TNF $\alpha$. By decreasing the number of activated microglial cells, nicotine and TNF $\alpha$ protects dopaminergic neurons of neurotoxin-treated mice (39). According to Cormier et al. (40), nicotine can protect brain mitochondria of rats against oxidative stress. by decreasing superoxide anion generation. Nicotine was also shown to compete with the NADH, H+ on the complex I of the electron transfer chain and to decrease the oxygen consumption, thereby protecting a central nervous system, which is relatively poor in antioxidant defense systems (41).

Studies in which nicotine was administered to patients with PD reported noticeable symptom improvement in the following areas: tremors, rigidity, disorganized thinking, bradykinesia, and depression (21, 42-43). However, the symptom relief observed was only temporary; the extended exposure to nicotine caused the nAChRs to desensitize, thus resulting in nicotine tolerance.

\section{Alzheimer's disease}

Alzheimer's disease is a form of dementia associated with neuronal loss, extracellular accumulation of amyloid $\beta$ peptide $(\mathrm{A} \beta)$, and intracellular deposits of tau protein. The activation of $\mathrm{nAChRs}$ with nicotine may exert a protective effect against $A \beta$ toxicity, which is one of the major factors contributing to the neurodegeneration observed in $\mathrm{AD}$ (44). 
Subcutaneous administration of nicotine to patients with $\mathrm{AD}$ improves visual attention, reaction time and perception (45). Nicotine may also antagonize the negative effects of anticholinergic drugs on rapid information processing tasks in humans and enhances cognitive performance in animals (42).

Results from current human population studies are inconclusive since cigarette smoking has been linked to both an increased and decreased risk for Alzheimer's. The complexity of this problem stems from a decrease in the participation of elderly smokers in case-controlled and cohort studies, which has created a survivor bias due to premature death (46). Although it has been suggested that nicotine and some agonists of nAChRs have neuroprotective effects, other substances in tobacco smoke such as lead and cadmium may actually cause cognitive deterioration. Furthermore, impaired lung function, a common adverse effect of cigarette smoking, has also been shown to be a risk factor for cognitive decline (46). Other studies have shown that chronic exposure to cigarette smoke may lead to an increase in $A \beta$ isoforms and abnormal tau phosphorylation. Therefore, smoking may facilitate the onset of AD, serving as a precipitating or causative factor in the development of the disease (47). In the Cochrane review by López-Arrieta et al. (48) the authors were not able to provide any evidence that nicotine is a useful treatment for Alzheimer's disease due to the poor quality of clinical trials.

\section{Role in schizophrenia and psychiatric diseases}

It is well documented, that the prevalence of smoking is very high among patients with schizophrenia (49). Among patients with schizophrenia, the odds of becoming a smoker are 5.3 times higher than in the general population (49). According to Dickerson et al. (50), about $64 \%$ of individuals with schizophrenia smoke, and according to de Leon and Diaz (49), it ranges from $44 \%$ for females to $71 \%$ for males.

It has been suggested, that the reason for the high prevalence of smoking among patients with schizophrenia may be related to the attempts to selfmedicate symptoms of the disease (cigarette smoking has been shown to modulate the symptoms of the disease). Another theory is that nicotine may decrease the side effects of the drugs used in patients with schizophrenia (51).

However, the self-medication theory has been largely criticized due to recent observational studies which have shown contrary findings: [1] no significant inverse relationship between smoking and symptoms of schizophrenia; [2] smoking precedes the onset of schizophrenia; and [3] a positive relationship between smoking and the intensity of schizophrenic symptoms (46). There is also some evidence linking genetic factors to the increased risk of both becoming a smoker and developing schizophrenia (50).

Current knowledge on the symptoms of schizophrenia stems from the dopamine hypothesis of schizophrenia. This theory postulates that an increased dopamine transmission in the mesolimbic pathway is associated with the "positive symptoms" of schizophrenia (delusions, hallucinations, and thought disorder). While a decrease in dopamine transmission in the mesocortical pathway is associated with the "negative symptoms" of schizophrenia (memory deficits, alogia, anhedonia, inattention) (52). Antipsychotics (dopamine antagonist) are the mainstay treatment for the positive symptoms of schizophrenia. However, effective options for negative symptoms are limited - a potential therapeutic area for nicotine. Since the negative symptoms of schizophrenia stems from hypodopaminergic transmission, nicotine could be beneficial in alleviating these symptoms due to its ability to increase dopamine (46). All nicotinic receptor subtypes that have a high affinity for nicotine, are located in these regions of the human brain, which are implicated in schizophrenia. Individuals suffering from schizophrenia may smoke to stimulate cortical dopaminergic activity, which is dissociated during this illness (51).

Antipsychotics, drugs used for the treatment of schizophrenia, autism, depression, attention deficit hyperactivity disorder, obsessive-compulsive disorder, and posttraumatic stress disorder. These valuable drugs very often also cause motor dysfunctions, which are the main side effects of the therapy. Nicotine and the agonists of nAChRs like varenicline have been found to decrease motor dysfunctions in animal models caused by antipsychotic drugs. (53-55).

Interestingly, nicotine has additional applications in schizophrenia. Since nAChRs are thought to be integral in memory processing, using an agonist such as nicotine could potentially improve a deteriorating memory - a known adverse effect of most antipsychotics (46). Many antipsychotics have been shown to inhibit the function of nAChRs, which further supports the hypothesis that these receptors might be involved in the memory impairment observed among patients taking antipsychotics (54). Also, animal models involving nicotine and the nAChR agonists were shown to mitigate antipsychotic-induced tardive dyskinesia (a common side effect of antipsychotic therapy) (54). There have 
also been studies showing nicotine may alleviate other extrapyramidal side effects (akathisia, dystonia) from antipsychotics, but current epidemiological studies are inconclusive (46). Concurrently to findings from $\mathrm{Xu}$ et al. (56), there is no significant association between cigarette smoking and the severity of psychiatric symptoms in other studies $(50,57)$.

\section{Role in movement disorders}

Preclinical analysis shows that agonists of nAChRs may improve drug-induced impairment of movement (54). It has been reported, that nAChR agonist, like nicotine and varenicline, reduce Ldopa-induced abnormal involuntary movements disorders in parkinsonian nonhuman primates and rodents. Moreover, nicotine decreases antipsychoticinduced abnormal involuntary movements in rodent models with tardive dyskinesia and attenuates dyskinetic symptoms of Tourette's syndrome (54).

L-dopa is a standard treatment for Parkinson's disease, but its side effects include dyskinesias. Investigation on L-dopa-induced dyskinesias (LIDs) was conducted in MPTP-lesioned monkeys. Administration of nicotine to L-dopa-treated monkeys led to decrease in LIDs (58). This probably happens through the mechanism of nAChRs' desensitization, as the nAChR antagonist mecamylamine reduces LIDs in parkinsonian rats to a similar extent and treatment of nicotine via minipump, that involves desensitization, leads to a similar attenuation of LIDs (59).

The effect of nicotine on the syndromes of ataxia has gained more attention, as both preclinical and clinical studies support the idea that it may improve components of ataxia (54) and this process involves nAChR's receptors. In a laboratory animals model involving olivocerebellar degeneration, nicotine could partially restore deficits in coordination, balance, and gait. Authors indicate these actions could be attributed to a nicotinic receptor-mediated effect unrelated to possible stimulant properties of the compound. It may be possible, that treatment with nicotine leads to the anti-ataxic effects, by direct alteration of the cellular activity of one or several structures within the cerebellar thalamocortical pathway (60).

\section{Role in Tourette's syndrome}

Tourette's syndrome is a movement disorder that is thought to result from abnormalities in the basal ganglia and is often treated with drugs that have antidopaminergic properties, such as the antipsychotic haloperidol (61). Nicotine has been found to allay the adverse effects of haloperidol, such as muscle weakness and locomotor hyperactivity. Nicotine has also been shown to decrease antipsychotic-induced involuntary movements in animal models with tardive dyskinesia and attenuate other dyskinetic symptoms of Tourette's syndrome (54).

Another hypothesis as to why nicotine might attenuate the occurrence and severity of symptoms in Tourette's is based on the ability of nicotine to cause receptor desensitization. It is believed that receptor desensitization reduces dopamine levels on striatal dopamine terminals and modulates the inhibitory cortical pathway, leading to attenuation of tics $(54,62)$. It is key to note that in addition to its agonistic properties nicotine can act as an antagonist. Several studies have shown that continued nicotine exposure helped mitigate some symptoms of Tourette's syndrome due to its antagonistic properties (61). Essentially, the continued exposure to nicotine will cause receptor desensitization while the antagonistic features allowed for continual symptom alleviation. Therefore, it has been proposed that a nicotinic receptor antagonist, such as mecamylamine, may have a potential role in managing the symptoms of Tourette's (61).

\section{Role in pain management therapy}

Based on recent studies, there is a consensus notion that nicotine reduces pain sensitivity in smokers and non-smokers (63).

The analgesic effect of nicotine seems to be due to the agonistic interaction with nAChRs. Some evidence to support this idea stems from the analgesic alkaloid epibatidine $(64,65)$. The high affinity of this alkaloid to the nicotinic receptors is associated with 200-fold greater potency and equal efficacy compared with morphine and is recognized to be the most potent agonist at several nicotinic receptor subtypes. Additionally, some analogs of epibatidine are potent agonists of nAChRs and have been found to have strong analgesic effects (65).

The activation of nAChRs is associated with improvement in differential inflammatory and neuropathic pain models involving mechanical, thermal or chemical stimulus (66).

Simons et al. (67) showed that exposure of rats to tobacco smoke may reduce perception of pain. In order to find if the analgesia is mediated via nicotinic receptors or also via $\mu$-opioid receptors, the rats were given mecamylamine, as nicotinic receptor antagonist and naltrexone an opiate antagonist. The antinociceptive effect was mediated by both types of receptors. 
A recent meta-analysis indicated that perioperative nicotine administration was associated with a statistically significant reduction in cumulative opioid consumption at $24 \mathrm{~h}$ and pain ratings (68). Therefore, nicotine is a potential adjunct to opioids and can help reduce opioid doses and opioid-related side effects (constipation, drowsiness, tolerance). It has been proposed that nicotine may act synergistically with morphine at spinal and supra-spinal levels because the activation of nicotinic receptors at this site seems to occur with $\mu$ - and $\varepsilon$ - opioid receptors $(65,69)$.

Moreover, nicotine stimulates the production of $\beta$-endorphins, that act synergistically to morphine. The antianalgesic role of nicotine may also be realized in an indirect way, by activating endorphins and promoting interaction between $\beta$-endorphins and morphine, when nicotine and morphine are applied simultaneously. Results presented by Simson et al. (67), indicating a contribution of opiate receptors in tobacco smoke-induced analgesia, are consistent with other findings that there is a cross-tolerance between nicotine and morphine and that repeated administration of both drugs attenuates the antinociceptive effect of both administered separately or simultaneously (70).

The reports on nicotine's analgesic properties are also in contrary to findings from Baiamonte et al. (71), who indicated that nicotine dependence produces hyperalgesia resulting in increases in nociceptive sensitivity, especially thermal hyperalgesia. However, the experiment was conducted on rats that were nicotine dependent. Authors attribute the thermal hyperalgesia effects to nicotine withdrawal. This finding contrasts with reports of increased pain during acute withdrawal (72). It is important to underline, that in nicotine-tolerant mice, the analgesia in response to morphine may be reduced (70). It should be pointed out that analgesic benefits might be limited to non-smokers due to the receptor desensitization and withdrawal effects observed in smokers (63). As such, nicotine may be an option for perioperative patients that are nicotine nad've as opposed to nicotine experienced patients.

\section{Role in ulcerative colitis}

Ulcerative colitis is a chronic disease characterized by inflammation that starts at the end region of the colon and progresses towards the small intestines (73). Recent studies suggest UC is a disease that affects mostly ex-smokers and non-smokers. Interestingly, these studies also theorized that smoking may protect against, help alleviate, and manage the symptoms of UC (73). This theory might hold some validity since ex-smokers have been observed to exhibit a delayed onset of UC compared to nonsmokers. It is interesting to note that this phenomenon is only observed in males. Nicotine use seems to be a potential option for maintaining UC remission, even more so than oral corticosteroids. However, this effect is not observed with active UC. The exact mechanism by which nicotine achieves its therapeutic effect in UC is unclear, but several theories have been proposed to explain the effect, these include production of oxygen free radicals, cytokines, eicosanoid-mediated inflammation, nitric oxide release, and reducing circular muscle activity (7374). Although many different formulations have been studied, only the patch was found to be efficacious in reducing symptoms or preventing recurrence of UC compared to traditional treatments such as prednisone or mesalamine (73). One caveat of this study is that the effectiveness of nicotine in the treatment of ulcerative colitis could not be fully evaluated due to a significant number of adverse events related to nicotine use among participants involved in the clinical trial (75).

\section{DISCUSSION/CONCLUSION}

Nicotine is potentially beneficial in various diseases; however, possible therapeutic applications of nicotine have been limited by both intrinsic and extrinsic factors. Intrinsically, nAChR receptor desensitization possess a tolerance problem: how do you sustain the therapeutic effect of nicotine over time without constant dose escalation? Also, how do you avoid the high addiction risk nicotine presents? Extrinsically, nicotine related research funding is sparse due to the stigma associated with nicotine and smoking.

The therapeutic potential of nicotine has been overshadowed by the devastating health consequences associated with tobacco products and the various compounds contained within it. The introduction of state-of-the-art therapeutic nicotine delivery systems containing pure nicotine, in recent years has given researchers a chance to better study the role of nicotine in treating chronic diseases. The use of pure nicotine will allow a user to bypass other carcinogenic compounds released from smokeless and combustible tobacco products and, thus, mitigate its toxicity. It is important to remember that the use of nicotine is often associated with people struggling to overcome addiction, dependence, and withdrawal. These cases further require the need to develop therapeutic nicotine delivery systems that are safe, effective, and titratable. As such, caution should always be exercised when implementing or administering nicotine as a new treatment option for diseases. Although our 
paper provides a comprehensive overview of the current literature pertaining to the therapeutic potential of nicotine, most of these studies are inconclusive and present an area of future research.

As more extensive nicotine-related studies are conducted, nicotine could have a place in the pharmacotherapy of various disease states. Future research should address whether it is possible to obtain similar pharmacological outcomes between fixed and variable (self-titration) nicotine administration. Unfortunately, there is currently no better system to deliver and to control the amount of nicotine inhaled other than combustible cigarettes. Fast nicotine delivery to the brain allows smokers to deliver the amount of nicotine needed to stop their cravings while avoiding toxic levels. The electronic cigarette is currently the only product in the market that allows users to adjust the amount of nicotine inhaled, which provides the patient a wide range of nicotine doses. Therefore, any device chosen or developed for treatment should have similar delivery characteristics to electronic cigarettes but each dose should be consistent.

Finally, there is currently a plethora of scientific data on the negative effects of smoking tobacco, but we lack adequate information on the potential therapeutic benefits of nicotine. As more empirical evidence for therapeutic uses of nicotine becomes available, we predict that pharmaceutical industries will divert their efforts and resources towards investigation and synthesis of novel nicotine delivery method for therapeutic purposes.

\section{Study limitations}

Due to the variability of research models described in the literature and the fact that some observations were done after former unspecified exposition to nicotine, our work does not suggest any dosages of nicotine that should be implemented in patients for therapeutical purposes. The work serves as a review of the available data and in many cases shows preclinical attempts that cannot be directly implemented in human.

\section{Conflict of interest}

The authors have no conflict of interest to report.

\section{REFERENCES}

1. Benowitz N.L.: Prim. Care 26, 611 (1999).

2. Benowitz N.L.: Annu. Rev. Pharmacol. Toxicol. 49, 57 (2009).
3. Changeux J.P., Bertrand D., Corringer P.J., Dehaene S., Edelstein S. et al.: Brain Res. Brain Res. Rev. 26, 198 (1998).

4. Changeux J.P.: Harvey Lect. 75, 85 (1981).

5. Changeux J.P.: C. R. Biol. 332, 421 (2009).

6. Benowitz N.L., Hukkanen J. Jacob P. 3rd.: Handb. Exp. Pharmacol. 192, 29 (2009).

7. http://www.ebi.ac.uk/chebi/searchId.do?chebiId=CHEBI:18723(accessed on 30.10.2017).

8. Feyerabend C., Ings R.M., Russel M.A.: Br. J. Clin. Pharmacol. 19, 239 (1985).

9. Raunio H., Rautio A., Gullstén H., Pelkonen O.: Br. J. Clin. Pharmacol. 52, 357 (2001).

10. Hukkanen J., Jacob P. 3rd., Benowitz N.L.: Pharmacol. Rev. 57, 79 (2005).

11. Jha P., Ramasundarahettige C., Landsman V., Rostron B., Thun M. et al.: N. Engl. J. Med. 368, 341 (2013).

12. Chang R.C., Ho Y.S., Wong S.,Gentleman S.M., Ng H.K.: Acta Neuropathol. 127, 53 (2014).

13. Dhalwani N.N., Szatkowski L., Coleman T., Fiaschi L., Tata L.J.: Pediatrics 135, 859 (2015).

14. Harrell P.T., Simmons V.N., Correa J.B., Padhya T.A., Brandon T.H.: Otolaryngol. Head Neck Surg. 151, 381 (2014).

15. Moore D., Aveyard P., Connock M., Wang D., Fry-Smith A., Barton P.: BMJ 338, 1024 (2009).

16. Shahab L., Brose L.S., West R.: CNS Drugs 27, 1007 (2013).

17. McClure J.B., Swan G.E.: CNS Drugs 20, 281 (2006).

18. Cahill K., Stevens S., Perera R., Lancaster T.: Cochrane Database Syst. Rev. 5 (2013).

19. Stead L.F., Perera R., Bullen C., Mant D., Hartmann-Boyce J. et al.: Cochrane Database Syst. Rev. 11 (2012).

20. Cahill K., Stevens S., Perera R., Lancaster T.: Cochrane Database Syst. Rev. 5 (2013).

21. Quik M., O’Leary K., Tanner C.M.: Mov. Disord. 23, 1641 (2008).

22. Sherer T.B., Betarbet R., Greenamyre J.T.: Neuroscientist 8, 192 (2002).

23. Hwang O.: Exp. Neurobiol. 22, 11 (2013).

24. Dunnett S.B., Bjorklund A.: Nature 399, 32 (1999).

25. Shimohama S., Sawada H., Kitamura Y., Taniguchi T.: Trends Mol. Med. 9, 360 (2003).

26. Barreto G.E., Iarkov A., Moran V.E.: Front Aging Neurosci. 6, 340 (2014).

27. Chen H., Huang X., Guo X., Mailman R.B., Park Y., Kamel F. et al.: Neurology 74, 878 (2010). 
28. De Reuck J., De Weweire M., Van Maele G., Santens P.: J. Neurol. Sci. 231, 35 (2005).

29. Fratiglioni L., Wang H.X.: Behav. Brain. Res. 113, 117 (2000).

30. Gorell J.M., Rybicki B.A., Cole Johnson C., Peterson E.L.: Neuroepidemiology 18, 303 (1999).

31. Grandinetti A., Morens D.M., Reed D., MacEachern D.: Am. J. Epidemiol. 139, 1129 (1994).

32. Morens D.M., Grandinetti A., Reed D., White L.R., Ross G.W.: Neurology 45, 1041 (1995).

33. Quik M., Perez X.A., Bordia T.: Mov. Disord. 27, 947 (2012).

34. Williams E., Linert W.: In Vivo 18, 391 (2004).

35. Shimohama S.: Biol. Pharm. Bull. 32, 332 (2009).

36. Bridge M.H.: PhD Thesis. Nicotine and the Fenton reaction, its interaction with iron(II) and iron(III) and the iron sulphur containing enzyme aconitase. TU-Vienna (2002).

37. Quik M., Jeyarasasingam G.: Eur. J. Pharmacol. 393, 223 (2000).

38. Takeuchi H., Yanagida T., Inden M., Takata K., Kitamura Y. et al.: J. Neurosci. Res. 87, 576 (2009).

39. Park H.J., Lee P.H., Ahn Y.W., Choi Y.J., Lee G. et al.: Eur. J. Neurosci. 26, 79 (2007).

40. Cormier A., Morin C., Zini R., Tillement J.P., Lagrue G.: Neuropharmacology 44, 642 (2003).

41. Fiskum G., Murphy A.N., Beal M.F.: J. Cereb. Blood Flow Metab. 19, 351 (1999).

42. Mihailescu S., Drucker-Colín R.: Acta Pharmacol. Sin. 21, 97 (2000).

43. Smucny J., Tregellas J.: Biochem. Pharmacol. 86, 1163 (2013).

44. Lombardo S., Maskos U.: Neuropharmacology 96, 255 (2015).

45. Jones G.M., Sahakian B.J., Levy R., Warburton D.M., Gray J.A.: Psychopharmacology (Berl.) 108, 485 (1992).

46. Dome P., Lazary J., Kalapos M.P., Rihmer Z.: Neurosci. Biobehav. Rev. 34, 295 (2010).

47. Durazzo T.C., Mattsson N., Weiner M.W.: Alzheimers Dement. 10, 122 (2014).

48. López-Arrieta J.M., Rodríguez J.L., Sanz F.: Cochrane Database Syst. Rev. 2 (2001).

49. de Leon J., Diaz F.J.: Schizophr. Res. 76, 135 (2005).

50. Dickerson F., Stallings C.R., Origoni A.E., Vaughan C., Khushalani S. et al.: Psychiatr. Serv. 64, 44 (2013).

51. Dalack G.W., Healy D.J., Meador-Woodruff J.H.: Am. J. Psychiatry 155, 1490 (1998).
52. Toda M., Abi-Dargham A.: Curr Psychiatry Rep. 9, 329 (2007).

53. Bordia T., McIntosh J.M., Quik M.: J. Pharmacol. Exp. Ther. 340, 612 (2012).

54. Quik M., Zhang D., Perez X.A., Bordia T.: Pharmacol. Ther. 144, 50 (2014).

55. Dawe S., Gerada C., Russell M.A., Gray J.A.: Psychopharmacology (Berl.) 117, 110 (1999).

56. Xu Y.M., Chen H.H., Li F., Deng F., Liu X.B. et al.: PLoS One 9, e88478 (2014).

57. Wijesundera H., Hanwella R., de Silva V.A.: Ann. Gen. Psychiatry 13, 7 (2014).

58. Quik M., Cox H., Parameswaran N., O'Leary K., Langston J.W. et al.: Ann. Neurol. 62, 588 (2007).

59. Bordia T., Campos C., McIntosh J.M., Quik M.: J. Pharmacol. Exp. Ther. 333, 929 (2010).

60. Wecker L., Engberg M.E., Philpot R.M., Lambert C.S., Kang C.W. et al. Neuropharmacology 73, 75 (2013).

61. Sanberg P.R., Vindrola-Padros C., Shytle R.D.: Physiol. Behav. 107, 801 (2012).

62. Orth M., Amann B., Robertson M.M., Rothwell J.C.: Brain 128, 1292 (2005).

63. Shi Y., Weingarten T.N., Mantilla C.B., Hooten W.M., Warner D.O.: Anesthesiology 113, 977 (2010).

64. Daly J.W., Spande T.F., Garraffo H.M.: J. Nat. Prod. 68, 1556 (2005).

65. Dineley K.T., Pandya A.A., Yakel J.L.: Trends Pharmacol. Sci. 36, 96 (2015).

66. Flores C.M.: Pain 88, 1 (2000).

67. Simons C.T., Cuellar J.M., Moore J.A., Pinkerton K.E., Uyeminami D. et al.: Neurosci. Lett. 389, 71 (2005).

68. Mishriky B.M., Habib A.S.: Anesth. Analg. 119, 268 (2013).

69. Suh H.W., Song D.K., Choi S.R., Chung K.M., Kim Y.H.: Neuropeptides 30, 479 (1996).

70. Zarrindast M.R., Khoshayand M.R., Shafaghi B.: Eur. Neuropsychopharmacol. 9, 227 (1999).

71. Baiamonte B.A., Valenza M., Roltsch E.A., Whitaker A.M., Baynes B.B. et al.: Neuropharmacology 77, 217 (2014).

72. Anderson K.L., Pinkerton K.E., Uyeminami D., Simons C.T., Carstens M.I., Carstens E.: Neurosci. Lett. 366, 86 (2004).

73. Lunney P.C., Leong R.W.: Aliment. Pharmacol. Ther. 36, 997 (2012).

74. Aldhous M.C., Prescott R.J., Roberts S., Samuel K., Waterfall M., Satsangi J.: Inflamm. Bowel. Dis. 14, 1469 (2008).

75. Slyk M.P., Frazee L.A.: Am. J. Ther. 4, 141 (1997).

Received: 29.09. 2017 